\title{
The Modulus of Nearly Uniform Smoothness in Orlicz Sequence Spaces
}

\author{
Shaoyong Zhang $(\mathbb{D}$, Meiling Zhang, and Yujia Zhan \\ Department of Mathematics, Harbin University of Science and Technology, Harbin 150080, China \\ Correspondence should be addressed to Shaoyong Zhang; 13904603565@139.com
}

Received 19 March 2019; Revised 10 June 2019; Accepted 7 July 2019; Published 22 July 2019

Academic Editor: Giuseppe Marino

Copyright (c) 2019 Shaoyong Zhang et al. This is an open access article distributed under the Creative Commons Attribution License, which permits unrestricted use, distribution, and reproduction in any medium, provided the original work is properly cited.

\begin{abstract}
It is well known that the modulus of nearly uniform smoothness related with the fixed point property is important in Banach spaces. In this paper, we prove that the modulus of nearly uniform smoothness in Köthe sequence spaces without absolutely continuous norm is $\Gamma_{X}(t)=t$. Meanwhile, the formula of the modulus of nearly uniform smoothness in Orlicz sequence spaces equipped with the Luxemburg norm is given. As a corollary, we get a criterion for nearly uniform smoothness of Orlicz sequence spaces equipped with the Luxemburg norm. Finally, the equivalent conditions of $R\left(a, l_{(\Phi)}\right)<1+a$ and $R W\left(a, l_{(\Phi)}\right)<1+a$ are given.
\end{abstract}

\section{Introduction}

In last century, the fixed point property has been studied by many scholars. A Banach space $X$ is said to have the fixed point property (FPP, for short) if every nonexpansive mapping $T: C \longrightarrow C$

$$
\|T x-T y\| \leq\|x-y\|, \quad \forall x, y \in C
$$

acting on a nonempty bounded closed and convex subset $C$ of $X$ has a fixed point. A natural generalization of FPP is the weak fixed point property (WFPP, for short). A Banach space $X$ is said to have the WFPP, whenever it satisfies the above condition from the definition of FPP with "weakly compact" in place of "bounded closed". In 1965 Kirk [1] proved that any reflexive Banach space with normal structure has the FPP and he also asserts that a Banach space with weak normal structure has WFPP. In this connection, see also the paper [2, 3]. In 1989 Prus [4] introduced a property of a Banach space $X$, called nearly uniform smoothness and he got that a Banach space is nearly uniform convex if and only if its dual space $X^{*}$ is nearly uniformly smooth. In paper [5], it is proved that weak nearly uniformly smooth Banach spaces have the FPP, which strengthens the result by S. Prus [6]. In [7], Domínguez-Benavides proved an important result on the existence of fixed points for nonexpansive mappings. In order to do this, he defined, for a Banach space $X$ and a nonnegative real number $a$, the parameter

$$
R(a, X):=\sup \left\{\liminf _{n \longrightarrow \infty}\left\|x_{n}+x\right\|\right\},
$$

where the supremum is taken over all $x \in X$ with $\|x\| \leq$ $a$ and all weakly null sequences $\left(x_{n}\right)$ in $B(X)$ such that $D\left[\left(x_{n}\right)\right]:=\lim \sup _{n \rightarrow \infty, m \rightarrow \infty}\left\|x_{n}-x_{m}\right\| \leq 1$. He also defined the coefficient

$$
M(X):=\sup \left\{\frac{1+a}{R(a, X)}: a \geq 0\right\} .
$$

The result that a Banach space $X$ has the WFPP, whenever $M(X)>1$ was obtained in [7] using an embedding into $l_{\infty}(X) / c_{0}(X)$.

In this paper we prove that $\Gamma_{X}(t)=t$ in Köthe sequence spaces without absolutely continuous norm. Furthermore, the formula of the modulus of nearly uniform smoothness in Orlicz sequence spaces equipped with the Luxemburg norm is given. We find the criteria for nearly uniform smoothness of Orlicz sequence spaces equipped with the Luxemburg. Finally, we prove that the condition of $R\left(a, l_{(\Phi)}\right)<1+a$ is equivalent to $\Phi \in \delta_{2} . R W\left(a, l_{(\Phi)}\right)=\Gamma_{l_{(\Phi)}}(a)$ and $R W\left(a, l_{(\Phi)}\right)<$ $1+a$ if and only if exist $\delta>0$ and $u_{0}>0$ such that $\Phi(u /(1+$ $a)) \leq((1-\delta) /(1+a)) \Phi(u)$ or $\Phi(u / a) \geq((1-\delta) / a) \Phi(u)$ when $|u| \leq u_{0}$. 


\section{Preliminaries}

Throughout this paper, $X$ is a Banach space which is assumed not to have the Schur property, in which $X$ has a weakly convergent sequence that is not norm convergent. By $S(X)$ and $B(X)$ we denote, respectively, the unit sphere and the unit ball of $X$, and by $l^{0}$ we denote the set of all real sequences.

Inspiring on the underlying ideas in the definition of nearly uniformly smooth spaces given by Prus [4] as well as on the modulus of uniform smoothness, in [8] DomínguezBenavides defined the modulus of nearly uniform smoothness.

Definition 1. The modulus of nearly uniform smoothness is defined by

$$
\Gamma_{X}(t):=\sup \left\{\inf _{n>1}\left(\frac{\left\|x_{1}+t x_{n}\right\|+\left\|x_{1}-t x_{n}\right\|}{2}-1\right)\right\},
$$

where the supremum is taken over all basic sequences $\left(x_{n}\right)$ in $B(X)$.

Definition 2. For each $0<a \leq 1$, we define

$$
\begin{aligned}
& R W(a, X):=\sup \left\{\left(\liminf _{n \longrightarrow \infty}\left\|x_{n}+x\right\|\right)\right. \\
& \quad \wedge\left(\liminf _{n \longrightarrow \infty}\left\|x_{n}-x\right\|\right):\left\{x_{n}\right\} \in B(X), x_{n} \stackrel{\omega}{\rightarrow} 0,\|x\| \\
& \quad \leq a\} .
\end{aligned}
$$

In [9], they have the important result that if a Banach space $X$ is reflexive and $R W(a, X)<1+a$ for some $a>0$ then $\Gamma_{X}^{\prime}(0)<1$ that the Banach space $X$ has the FPP.

They use the above result to give a direct proof that the uniformly nonsequare Banach space has the FPP.

In paper [8] it is shown that a Banach space $X$ is nearly uniformly smooth if and only if $X$ is reflexive and

$$
\Gamma_{X}^{\prime}(0):=\lim _{t \rightarrow 0^{+}} \frac{\Gamma_{X}(t)}{t}=0
$$

and

$$
\Gamma_{X}(t):=\sup \left\{\inf _{n>1}\left(\frac{\left\|x_{1}+t x_{n}\right\|+\left\|x_{1}-t x_{n}\right\|}{2}-1\right)\right\},
$$

where the supremum is taken over all weak null sequences $\left(x_{n}\right)$ in $B(X)$.

The following theorem was proved in [10].

Theorem 3. If $X$ is a reflexive Banach space and $\Gamma^{\prime}{ }_{X}(0)<1 / 2$, $X$ has the FPP.

Definition 4. A Banach space $X$ is called a Köthe sequence space if for every $x \in l^{0}$ and $y \in X$ satisfying $|x(i)| \leq|y(i)|$ for all $i \in N$, we have $x \in X$ and $\|x\| \leq\|y\|$ and if there is a $x=(x(i)) \subset X$ with $x(i)>0$ for all $i \in N($ see $[11,12])$. Let

$$
\begin{aligned}
X_{a} & :=\{x \\
& \in X: \lim _{n \rightarrow \infty}\|(0,0, \ldots 0, x(n+1), x(n+2), \ldots)\| \\
& =0\} .
\end{aligned}
$$

A Köthe sequence space $X$ is said to be norm absolutely continuous if $X_{a}=X$.

Definition 5. A Köthe sequence space $X$ is said to have the semi-Fatou property if for every sequence $\left\{x_{n}\right\} \subset X$ and $x \in$ $X$ satisfying $\left|x_{n}(i)\right| \uparrow|x(i)|$ for all $i \in N$ there holds $\left\|x_{n}\right\| \longrightarrow$ $\|x\|$.

Definition 6. $\Phi: R \longrightarrow R_{+}$is called an Orlicz function if it has the following properties:

(1) $\Phi$ is even, continuous, convex and $\Phi(0)=0$;

(2) $\Phi(u)>0$ for all $u \neq 0$.

Definition 7. For every Orlicz function $\Phi$ we define the complementary function $\Psi: R \longrightarrow[0, \infty]$ by the formula

$$
\Psi(u):=\sup _{u>0}\{u|v|-\Phi(u)\}
$$

for every $v \in R$.

Definition 8. We say that $\Phi$ satisfies the $\delta_{2}$-condition $\left(\Phi \in \delta_{2}\right.$ for short) if there exist $k>0, u_{0}>0$ such that the inequality

$$
\Phi(2 u) \leq k \Phi(u)
$$

whenever $|u| \leq u_{0}$.

We say that $\Phi$ satisfies the $\overline{\delta_{2}}$-condition $\left(\Phi \in \overline{\delta_{2}}\right.$ for short $)$ if its complementary function $\Psi$ satisfies the $\delta_{2}$-condition.

The Orlicz sequence space $l_{(\Phi)}$ is defined to be the set $\{x \in$ $l^{0}: I_{\Phi}(\lambda x)=\sum_{i=1}^{\infty} \Phi(\lambda x(i))<\infty$ for some $\left.\lambda>0\right\}$ equipped with the Luxemburg norm

$$
\|x\|_{(\Phi)}:=\inf \left\{k>0: I_{\Phi}\left(\frac{x}{k}\right) \leq 1\right\} .
$$

To simplify notations, we put $l_{(\Phi)}=\left(l_{\Phi},\|\cdot\|_{(\Phi)}\right)$.

The basic informations on Orlicz spaces can be found in [13-15].

\section{Main Results}

Theorem 9. Let $X$ be a Köthe sequence space with the SemiFatou property. If the norm of $X$ is not absolutely continuous, $\Gamma_{X}(t)=t$.

Proof. Suppose that the norm of $X$ is not absolutely continuous. Then there exist $\varepsilon_{0}>0$ and $x_{0} \in S(X)$ such that

$$
\lim _{n \rightarrow \infty}\left\|\sum_{i=n+1}^{\infty} x_{0}(i) e_{i}\right\|=\varepsilon_{0} \text {, }
$$

where $e_{i}=(0,0, \ldots, \stackrel{i \text { th }}{1}, 0, \ldots)$. 
Take any $\varepsilon>0$. By $\lim _{n \rightarrow \infty}\left\|\sum_{i=n+1}^{\infty} x_{0}(i) e_{i}\right\|=\varepsilon_{0}$, there exists $n_{1} \in N$ such that

$$
\left\|\sum_{i=n_{1}}^{\infty} x_{0}(i) e_{i}\right\| \leq(1+\varepsilon) \varepsilon_{0} .
$$

Notice that

$$
\lim _{m \longrightarrow \infty}\left\|\sum_{i=n_{1}+1}^{m} x_{0}(i) e_{i}\right\|=\left\|\sum_{i=n_{1}+1}^{\infty} x_{0}(i) e_{i}\right\|,
$$

so there exists $n_{2}>n_{1}$ such that

$$
(1-\varepsilon) \varepsilon_{0} \leq\left\|\sum_{i=n_{1}+1}^{n_{2}} x_{0}(i) e_{i}\right\| \leq(1+\varepsilon) \varepsilon_{0} .
$$

In this way, we get a sequence $\left\{n_{i}\right\}$ of natural numbers such that

$$
(1-\varepsilon) \varepsilon_{0} \leq\left\|\sum_{j=n_{i}+1}^{n_{i+1}} x_{0}(i) e_{i}\right\| \leq(1+\varepsilon) \varepsilon_{0}, \quad i=1,2, \ldots
$$

Put $x_{i}=\sum_{j=n_{i}+1}^{n_{i+1}} x_{0}(j) e_{j}$ and $v_{k}=\sum_{j=n_{k}+1}^{\infty} x_{0}(j) e_{j}$. Then

(a) $\left\|x_{i}\right\| \longrightarrow \varepsilon_{0}$ as $i \longrightarrow \infty$;

(b) $x_{i} \stackrel{w}{\rightarrow} 0$ as $i \longrightarrow \infty$. It is well known that, for any Köthe space $X$, we have

$$
X^{*}=X^{\prime} \oplus S,
$$

where $S$ is the space of all singular functionals over $X . X^{\prime}=$ $\left\{y \in l^{0}\right.$ : the series $\sum_{i=1}^{\infty} x(i) y(i)$ converges for all $\left.x \in X\right\}$. This means that every $f \in X^{*}$ is uniquely represented in the form

$$
f=T_{y}+\varphi,
$$

where $\varphi \in S$ and for $y \in X^{\prime}$ the function $T_{y}$ is defined by

$$
T_{y}(x)=\sum_{i=1}^{\infty} x(i) y(i)
$$

for all $x \in X$.

Consider that the series $\sum_{i=1}^{\infty} x(i) y(i)$ converges. We have

$$
\lim _{n \longrightarrow \infty} \sum_{j=1}^{\infty} x_{n}(j) y(j)=\lim _{i \longrightarrow \infty} \sum_{j=n_{i}+1}^{n_{i+1}} x_{i}(j) y(j)=0 .
$$

(c) Put $z_{i}=v_{i} /\left\|v_{i}\right\|$ and $w_{k}=\left(x_{k}-x_{k+1}\right) /\left\|x_{k}-x_{k+1}\right\|$ for all $i, k \in N$. Then for $k>i$ the following holds:

$$
\begin{aligned}
& \frac{1}{2}\left(\left\|z_{i}+t w_{n}\right\|+\left\|z_{i}-t w_{n}\right\|\right) \\
& \quad=\frac{1}{2}\left(\left\|\frac{v_{i}}{\left\|v_{i}\right\|}+t \frac{x_{k}-x_{k+1}}{\left\|x_{k}-x_{k+1}\right\|}\right\|\right. \\
& \left.+\left\|\frac{v_{i}}{\left\|v_{i}\right\|}-t \frac{x_{k}-x_{k+1}}{\left\|x_{k}-x_{k+1}\right\|}\right\|\right) \\
& \geq \frac{1}{2}\left(\left\|\frac{x_{k}}{\left\|v_{i}\right\|}+t \frac{x_{k}}{\left\|x_{k}-x_{k+1}\right\|}\right\|\right. \\
& \left.+\left\|\frac{x_{k+1}}{\left\|v_{i}\right\|}+t \frac{x_{k+1}}{\left\|x_{k}-x_{k+1}\right\|}\right\|\right) \geq(1-\varepsilon) \varepsilon_{0}\left(\frac{1}{\left\|v_{i}\right\|}\right. \\
& \left.\quad+t \frac{1}{\left\|x_{k}-x_{k+1}\right\|}\right) \geq \frac{1-\varepsilon}{1+\varepsilon}(1+t) .
\end{aligned}
$$

By the arbitrariness of $\varepsilon$, we get $\Gamma_{X}(t) \geq t$. It is clear that $\Gamma_{X}(t) \leq t$. Therefore $\Gamma_{X}(t)=t$.

Theorem 9 also can be derived from the result of Hudzik and Mastyło [16].

Corollary 10. If $\Phi \notin \delta_{2}, \Gamma_{X}(t)=t$ for any $t>0$.

Proof. Since $\Phi \notin \delta_{2}$, we have that the norm of $l_{(\Phi)}$ is not absolutely continuous. Hence $\Gamma_{X}(t)=t$ for any $t>0$.

Theorem 11. Let $X$ be a reflexive Köthe sequence space and the norm of $X$ be absolutely continuous. Then

$$
\Gamma_{X}(t):=\sup \left\{\inf _{n>1}\left(\left\|x_{1}+t x_{n}\right\|-1\right)\right\},
$$

where the supremum is taken over all basic sequences $\left(x_{n}\right)$ in $S(X)$.

Proof. It is clear that if $\left\{x_{n}\right\}$ is a basic sequence in $B(X)$, $\left(x_{n} /\left\|x_{n}\right\|\right)$ is also a basic sequence in $S(X)$. We only prove that $\liminf _{n \rightarrow \infty}\left(\left\|x_{1}+t x_{n}\right\|-\left\|x_{1}-t x_{n}\right\|\right)=0$. For any $\varepsilon>0$, by the norm of $X$ which is absolutely continuous, there is a $i_{0} \in N$ such that

$$
\left\|\sum_{i=i_{0}+1}^{\infty} x_{1}(i) e_{i}\right\|<\varepsilon
$$

and since $\left\{x_{n}\right\}$ is a basic sequence, we have that the sequence $\left\{x_{n}\right\}$ converge to 0 in coordinate, and there exists a $n_{1} \in N$ such that

$$
\left\|\sum_{i=1}^{i_{0}} x_{n}(i) e_{i}\right\|<\varepsilon
$$


whenever $n>n_{1}$. So

$$
\begin{aligned}
& \|\| x_{1}+t x_{n}\|-\| x_{1}-t x_{n}\|\| \\
& \leq\left\|\sum_{i=1}^{i_{0}} x_{1}(i) e_{i}+t \sum_{i=i_{0}+1}^{\infty} x_{n}(i) e_{i}\right\| \\
& -\left\|\sum_{i=1}^{i_{0}} x_{1}(i) e_{i}-t \sum_{i=i_{0}+1}^{\infty} x_{n}(i) e_{i}\right\|+2 \varepsilon(1+t) .
\end{aligned}
$$

Noting that $X$ is a Köthe space, we have

$$
\begin{aligned}
& \left\|\sum_{i=1}^{i_{0}} x_{1}(i) e_{i}+t \sum_{i=i_{0}+1}^{\infty} x_{n}(i) e_{i}\right\| \\
& =\left\|\sum_{i=1}^{i_{0}} x_{1}(i) e_{i}-t \sum_{i=i_{0}+1}^{\infty} x_{n}(i) e_{i}\right\| .
\end{aligned}
$$

Therefore

$$
\left|\left\|x_{1}+t x_{n}\right\|-\left\|x_{1}-t x_{n}\right\|\right| \leq 2 \varepsilon(1+t)
$$

whenever $n>n_{1}$, that is, $\liminf _{n \rightarrow \infty}\left(\left\|x_{1}+t x_{n}\right\|-\left\|x_{1}-t x_{n}\right\|\right)=$ 0 .

In order to get the criterion that an Orlicz sequence space $l_{(\Phi)}$ is nearly uniformly smooth, we may assume that $\Phi \in \overline{\delta_{2}}$ and $\Psi \in \delta_{2}$ and that the space $l_{(\Phi)}$ is reflexive. Put $N(x)=\{i \in$ $N: x(i) \neq 0\}$. For any $x \in S\left(l_{(\Phi)}\right)$ with finite $N(x)$ and $t>0$, we define $c_{x, n}(t)$ as follows:

$$
\begin{gathered}
c_{x, n}(t):=\sup \left\{c_{x, y, t}>0: I_{\Phi}\left(\frac{x}{c_{x, y, t}}\right)+I_{\Phi}\left(\frac{t y}{c_{x, y, t}}\right)\right. \\
\left.=1 \text { for } y \in S\left(l_{(\Phi)}\right) \text { with } n \leq \min N(y)<\infty\right\} .
\end{gathered}
$$

It is clear that $\left\{c_{x, n}(t)\right\}_{n=1}^{\infty}$ is decreasing. Hence $c_{x}(t)=$ $\lim _{n \rightarrow \infty} c_{x, n}(t)$.

Theorem 12. Supposed that $\Phi \in \delta_{2}$ and $\Psi \in \delta_{2}$. For an Orlicz sequence space $l_{(\Phi)}$, there holds

$$
\begin{aligned}
\Gamma_{l_{(\Phi)}}(t):= & \sup \left\{c_{x}(t): x \in S\left(l_{(\Phi)}\right) \text { with finite } N(x)\right\} \\
& -1 .
\end{aligned}
$$

Proof. Let

$$
d_{\Phi}:=\sup \left\{c_{x}(t): x \in S\left(l_{(\Phi)}\right) \text { with finite } N(x)\right\} .
$$

Then for any $\varepsilon \in\left(0, d_{\Phi}\right)$, there exists $\|x\|_{(\Phi)}=1$ with finite $N(x)$ such that $c_{x}>d_{\Phi}-\varepsilon$. By the definition of $c_{x}(t)$, there exists $n_{1} \in N$ such that

$$
\begin{gathered}
\sup \left\{c_{x, y, t}>0: I_{\Phi}\left(\frac{x}{c_{x, y}}\right)+I_{\Phi}\left(\frac{t y}{c_{x, y}}\right)=1 \text { for } y\right. \\
\left.\in S\left(l_{(\Phi)}\right) \text { and } \min N(y) \geq n_{1}\right\}>d_{\Phi}-\varepsilon
\end{gathered}
$$

whenever $n \geq n_{1}$. By the definition of $c_{x}(t)$, there exists $y_{1} \epsilon$ $S\left(l_{(\Phi)}\right)$ with $\min N\left(y_{1}\right)>n_{1}$ such that $c_{x, y_{1}, t}>d_{\Phi}-\varepsilon$, that is to say,

$$
I_{\Phi}\left(\frac{x}{d_{\Phi}-\varepsilon}\right)+I_{\Phi}\left(\frac{t y_{1}}{d_{\Phi}-\varepsilon}\right)>1
$$

Hence there exists $n_{2}>n_{1}$ such that

$$
I_{\Phi}\left(\frac{x}{d_{\Phi}-\varepsilon}\right)+\sum_{i=n_{1}+1}^{n_{2}} \Phi_{i}\left(\frac{t y_{1}(i)}{d_{\Phi}-\varepsilon}\right)>1 .
$$

Since $n_{2}>n_{1}$, we also have

$$
\begin{array}{r}
\sup \left\{c_{x, y}>0: I_{\Phi}\left(\frac{x}{c_{x, y}}\right)+I_{\Phi}\left(\frac{t y}{c_{x, y}}\right)\right. \\
\left.=1 \text { and } \min N(y) \geq n_{2}\right\}>d_{\Phi}-\varepsilon
\end{array}
$$

and there exists $y_{2} \in l_{(\Phi)}$ with $\min N\left(y_{2}\right)>n_{1}$ such that $c_{x, y_{2}}>d_{\Phi}-\varepsilon$, that is to say,

$$
I_{\Phi}\left(\frac{x}{d_{\Phi}-\varepsilon}\right)+I_{\Phi}\left(\frac{t y_{2}}{d_{\Phi}-\varepsilon}\right)>1 .
$$

Hence there also exists $y_{2} \in S\left(l_{(\Phi)}\right)$ with $\min N\left(y_{2}\right)>$ $\max N\left(y_{1}\right)$ such that

$$
I_{\Phi}\left(\frac{x}{d_{\Phi}-\varepsilon}\right)+\sum_{i=n_{2}+1}^{n_{3}} \Phi_{i}\left(\frac{t y_{2}(i)}{d_{\Phi}-\varepsilon}\right)>1 .
$$

In such a way, there exist a sequence $\left\{y_{k}\right\}_{k=1}^{\infty} \subset S\left(l_{(\Phi)}\right)$ and a sequence of natural numbers $n_{1}<n_{2}<n_{3}<\cdots$ such that

$$
I_{\Phi}\left(\frac{x}{d_{\Phi}-\varepsilon}\right)+\sum_{i=n_{k}+1}^{n_{k+1}} \Phi\left(\frac{t y_{k}(i)}{d_{\Phi}-\varepsilon}\right)>1
$$

for all $k \in N$. It is clear that $y_{k} \stackrel{w}{\rightarrow} 0$.

For any $k>i_{0}$, we have

$$
\begin{aligned}
I_{\Phi}\left(\frac{x+t y_{k}}{d_{\Phi}-\varepsilon}\right) & =\sum_{i=n_{k}+1}^{n_{k+1}} \Phi_{i}\left(\frac{t y_{k}(i)}{d_{\Phi}-\varepsilon}\right)+I_{\Phi}\left(\frac{x(i)}{d_{\Phi}-\varepsilon}\right) \\
& >1,
\end{aligned}
$$

that is to say,

$$
\left\|x+t y_{k}\right\|_{(\Phi)}>d_{\Phi}-\varepsilon \text {. }
$$

Therefore $\Gamma_{l_{(\Phi)}}(t) \geq d_{\Phi}-\varepsilon-1$ and by the arbitrariness of $\varepsilon$, we have $\Gamma_{l_{(\Phi)}}(t) \geq d_{\Phi}-1$.

Now we prove that $\Gamma_{l_{(\Phi)}}(t) \leq d_{\Phi}-1$. By the definition of $d_{\Phi}$, we always have

$$
\begin{aligned}
& \lim _{n \longrightarrow \infty} \sup \left\{c_{x, y, t}>0: I_{\Phi}\left(\frac{x}{c_{x, y, t}}\right)+I_{\Phi}\left(\frac{t y}{c_{x, y, t}}\right)\right. \\
& \left.=1 \text { for } y \in S\left(l_{(\Phi)}\right) \text { and } \min N(y) \geq n\right\} \leq d_{\Phi}
\end{aligned}
$$

for any $x \in S\left(l_{\Phi}\right)$ with finite $N(x)$. 
Taking any weak null sequence $\left\{x_{n}\right\} \subset S\left(l_{(\Phi)}\right)$ and $\varepsilon>0$, by $\Phi \in \delta_{2}$ there exists $i_{0}>0$ such that $\left\|\sum_{i=i_{0}+1}^{\infty} x_{1}(i) e_{i}\right\|_{(\Phi)}<\varepsilon$. Since $x_{n} \stackrel{w}{\rightarrow} 0$, there is a $n_{0} \in N$ such that $\left\|\sum_{i=1}^{i_{0}} x_{n}(i) e_{i}\right\|_{(\Phi)}<$ $\varepsilon$ when $n \geq n_{0}$. Hence

$$
\begin{aligned}
& \left\|x_{1}+t x_{n}\right\|_{(\Phi)} \leq \| \sum_{i=1}^{i_{0}}\left(x_{1}(i)+t x_{n}(i)\right) e_{i} \\
& +\sum_{i=i_{0}+1}^{\infty}\left(x_{1}(i)+t x_{n}(i)\right) e_{i}\left\|_{(\Phi)} \leq\right\| \sum_{i=1}^{i_{0}} x_{1}(i) e_{i} \\
& +\sum_{i=i_{0}+1}^{\infty} t x_{n}(i) e_{i} \|_{(\Phi)}+(1+t) \varepsilon .
\end{aligned}
$$

We next estimate the $\left\|\sum_{i=1}^{i_{0}} x_{1}(i) e_{i}+\sum_{i=i_{0}+1}^{\infty} t x_{n}(i) e_{i}\right\|_{(\Phi)}$, when $n>n_{0}$. Put $z_{1}=\sum_{i=1}^{i_{0}} x_{1}(i) e_{i} /\left\|\sum_{i=1}^{i_{0}} x_{1}(i) e_{i}\right\|_{(\Phi)}$ and $z_{n}=$ $\sum_{i=i_{0}+1}^{\infty} x_{n}(i) e_{i} /\left\|\sum_{i=i_{0}+1}^{\infty} x_{n}(i) e_{i}\right\|_{(\Phi)}$ for $n>n_{0}$. There exists $n_{1} \geq n_{0}$ such that if $I_{\Phi}\left(z_{1} / c_{z_{1}, z_{n}, t}\right)+I_{\Phi}\left(t z_{n} / c_{z_{1}, z_{n}, t}\right)=1$, then $c_{z_{1}, z_{n}, t} \leq d_{\Phi}+\varepsilon$ for $n \geq n_{1}$.

$$
\begin{gathered}
I_{\Phi}\left(\frac{\sum_{i=1}^{i_{0}} z_{1}(i) e_{i}+\sum_{i=i_{0}+1}^{\infty} t z_{n}(i) e_{i}}{d_{\Phi}+\varepsilon}\right) \\
\quad=I_{\Phi}\left(\frac{z_{1}}{d_{\Phi}+\varepsilon}\right)+I_{\Phi}\left(\frac{t z_{n}}{d_{\Phi}+\varepsilon}\right) \\
\quad \leq I_{\Phi}\left(\frac{z_{1}}{c_{z_{1}, z_{n}, t}}\right)+I_{\Phi}\left(\frac{t z_{n}}{c_{z_{1}, z_{n}, t}}\right)=1,
\end{gathered}
$$

that is,

$$
\left\|\sum_{i=1}^{i_{0}} z_{1}(i) e_{i}+\sum_{i=i_{0}+1}^{\infty} t z_{n}(i) e_{i}\right\|_{(\Phi)} \leq d_{\Phi}+\varepsilon
$$

when $n \geq n_{0}$.

When $n \geq n_{1}$. It follows that

$$
\begin{aligned}
& \left\|\sum_{i=1}^{i_{0}} x(i) e_{i}-\sum_{i=i_{0}+1}^{\infty} x_{n}(i) e_{i}\right\|_{(\Phi)} \\
& \leq\|\| \sum_{i=1}^{i_{0}} x_{1}(i) e_{i} \|_{(\Phi)} \sum_{i=1}^{i_{0}} z_{1}(i) e_{i} \\
& \quad+\left\|\sum_{i=i_{0}+1}^{\infty} x_{n}(i) e_{i}\right\|_{(\Phi)} \sum_{i=i_{0}+1}^{\infty} t z_{n}(i) e_{i}\left\|_{(\Phi)} \leq\right\| \sum_{i=1}^{i_{0}} z_{1}(i) e_{i} \\
& \quad+\sum_{i=i_{0}+1}^{\infty} t z_{n}(i) e_{i} \|_{(\Phi)} \leq d_{\Phi}+\varepsilon .
\end{aligned}
$$

By the arbitrariness of $\varepsilon$, we get the inequality $\Gamma_{l_{(\Phi)}}(t) \leq d_{\Phi}-1$. Summing up, we obtain the equality $\Gamma_{l_{(\Phi)}}(t)=d_{\Phi}-1$.
Lemma 13. Let $\Phi \in \bar{\delta}_{2}$. Then for any $\varepsilon>0$ and $u_{0}>0$, there exists $\delta>0$ such that

$$
\Phi(t u) \leq t \varepsilon \Phi(u)
$$

whenever $|u| \leq \Phi^{-1}(1)$ and $t \in(0, \delta)$.

Proof. By $\Phi \in \bar{\delta}_{2}$, for any $u_{0}>0$, there exists $\theta \in(0,1)$ such that

$$
\Phi\left(\frac{u}{2}\right) \leq \frac{\theta}{2} \Phi(u)
$$

whenever $|u| \leq \Phi^{-1}(1)$.

For any $\varepsilon>0$, there is a $n \in N$ such that $\theta^{n}<\varepsilon$. Put $\delta=1 / 2^{n}$. Then

$$
\Phi(\delta u)=\Phi\left(\frac{u}{2^{n}}\right) \leq \frac{\theta^{n}}{2^{n}} \Phi(u) \leq \delta \varepsilon \Phi(u)
$$

whenever $|u| \leq \Phi^{-1}(1)$. Hence

$$
\begin{aligned}
\Phi(t u) & =\Phi\left(\frac{t}{\delta} \delta u\right) \leq \frac{t}{\delta} \Phi(\delta u) \leq \frac{t}{\delta} \delta \varepsilon \Phi(u) \\
& =t \varepsilon \Phi(u)
\end{aligned}
$$

whenever $t \in(0, \delta)$ and $|u| \leq \Phi^{-1}(1)$.

Corollary 14. An Orlicz sequence space $l_{(\Phi)}$ is nearly uniformly smooth if and only if $\Phi \in \delta_{2}$ and $\Phi \in \bar{\delta}_{2}$.

Proof. Since $\Phi \in \delta_{2}$ and $\Phi \in \bar{\delta}_{2}$ imply that the space $l_{(\Phi)}$ is reflexive, we only need to prove the sufficiency. For any $x \in$ $S\left(l_{\Phi}\right)$ with finite $N(x)$ that is $\max \{i: i \in N(x)\}<n$, and $y \in$ $S\left(l_{\Phi}\right)$ with $\min N(y) \geq n$. For any $\varepsilon>0$, by Lemma 13 , there exists $\delta>0$ such that $\Phi(t u) \leq t \varepsilon \Phi(u)$ when $|u| \leq \Phi^{-1}(1)$ and $t \in(0, \delta)$. Hence if $c_{x, y, t} \geq 1$ and $t \in(0, \delta)$

$$
\begin{aligned}
1 & =I_{\Phi}\left(\frac{x}{c_{x, y, t}}\right)+I_{\Phi}\left(\frac{t y}{c_{x, y, t}}\right) \\
& \leq \frac{1}{c_{x, y, t}} I_{\Phi}(x)+\frac{t \varepsilon}{c_{x, y, t}} I_{\Phi}(y)=\frac{1}{c_{x, y, t}}(1+t \varepsilon) .
\end{aligned}
$$

This shows that $c_{x, y, t} \leq 1+t \varepsilon$, that is, $\Gamma_{l_{(\Phi)}} \leq t \varepsilon$ when $t \in(0, \delta)$. Therefore $\Gamma_{l_{(\Phi)}}^{\prime}(0)=0$.

Corollary 15. Let $l_{p}(1<p<\infty)$ be a Lebesgue sequence space. Then $\Gamma_{l_{p}}(t)=\left(1+t^{p}\right)^{1 / p}-1$.

Proof. It is well known that the Lebesgue sequence space $l_{p}(1<p<\infty)$ is an Orlicz sequence space generated by $\Phi(u)=|u|^{p}$. For any $x, y \in S\left(l_{p}\right)$, we now consider the following:

$$
I_{\Phi}\left(\frac{x}{c_{x, y, t}}\right)+I_{\Phi}\left(\frac{t y}{c_{x, y, t}}\right)=1 .
$$


That is,

$$
\sum_{i=1}^{\infty}\left|\frac{x(i)}{c_{x, y, t}}\right|^{p}+\sum_{i=1}^{\infty}\left|\frac{t y(i)}{c_{x, y, t}}\right|^{p}=1
$$

Hence

$$
\frac{1}{c_{x, y, t}^{p}} \sum_{i=1}^{\infty}|x(i)|^{p}+\frac{t^{p}}{c_{x, y, t}^{p}} \sum_{i=1}^{\infty}|y(i)|^{p}=\frac{1+t^{p}}{c_{x, y, t}^{p}}=1,
$$

that is to say, $c_{x, y, t}=\left(1+t^{p}\right)^{1 / p}$. So $\Gamma_{l_{(p)}}(t)=\left(1+t^{p}\right)^{1 / p}-1$ for $t>0$.

It is also well known that if $\Phi$ is an Orlicz function, $\lim _{u \rightarrow 0}(\Phi(u) / u)=a$. If $a>0, l_{(\Phi)}$ has Schur property [17]

Theorem 16. Suppose that $\Phi \in \delta_{2}$ and $a \in(0,1)$. $\left.R W\left(a, l_{(\Phi)}\right)\right)<1+a$ if and only if exist $\delta>0$ and $u_{0}>0$ such that $\Phi(u /(1+a)) \leq((1-\delta) /(1+a)) \Phi(u)$ or $\Phi(u / a) \geq$ $((1+\delta) / a) \Phi(u)$ when $|u| \leq u_{0}$.

Proof.

Necessity. If there exists a sequence $u_{n} \searrow 0$ such that

$$
\Phi\left(\frac{u_{n}}{1+a}\right) \geq \frac{1-1 / n}{1+a} \Phi\left(u_{n}\right)
$$

and

$$
\Phi\left(\frac{u_{n}}{a}\right) \geq \frac{1+1 / n}{a} \Phi\left(u_{n}\right)
$$

for each $n \in N$, take $l_{n} \in N$ such that

$$
l_{n} \Phi\left(\frac{u_{n}}{1+a}\right) \leq 1<\left(l_{n}+1\right) \Phi\left(\frac{u_{n}}{1+a}\right) .
$$

Putting $x_{n}=(\overbrace{u_{n}, u_{n}, \ldots, u_{n}}^{l_{n}}, 0, \ldots)$, then $I_{\Phi}\left(x_{n} /(1+a)\right) \longrightarrow 1$. So $\left\|x_{n}\right\|_{(\Phi)} \longrightarrow 1+a$ as $n \longrightarrow \infty$.

$$
\begin{aligned}
\left\|x_{n}\right\|_{(\Phi)} & \leq I_{\Phi}\left(x_{n}\right)=l_{n} \Phi\left(u_{n}\right) \\
& \leq l_{n}\left(\frac{1+a}{1-1 / n} \Phi\left(\frac{u_{n}}{1+a}\right)\right) \leq \frac{1+a}{1-1 / n} .
\end{aligned}
$$

Therefore

$$
\lim _{n \longrightarrow \infty} I_{\Phi}\left(x_{n}\right)=1+a .
$$

Take $k_{n} \in N$ such that

$$
k_{n} \Phi\left(u_{n}\right) \leq 1<\left(k_{n}+1\right) \Phi\left(u_{n}\right) .
$$

It is clear that $k_{n}<l_{n}$ for $n$ large enough. Let $y_{1}^{(n)}=$ $(\overbrace{u_{n}, u_{n}}^{l_{n}-k_{n}}, \ldots, u_{n}, 0, \ldots)$

$$
\begin{gathered}
y_{2}^{(n)}=(\overbrace{0,0, \ldots, 0,}^{l_{n}}, \overbrace{u_{n}, u_{n}, \ldots, u_{n}}^{k_{n}}, 0, \ldots), \ldots, \\
\ldots \\
y_{m}^{(n)}=(\overbrace{0,0, \ldots, 0,}^{l_{n}+(m-2) k_{n}} \overbrace{u_{n}, u_{n}, \ldots, u_{n}}^{k_{n}}, 0, \ldots), \ldots
\end{gathered}
$$

Then $I_{\Phi}\left(y_{m}^{(n)}\right) \longrightarrow 1$ and $I_{\Phi}\left(y_{1}^{(n)}\right)=I_{\Phi}\left(x_{n}\right)-I_{\Phi}\left(y_{m}^{(n)}\right) \longrightarrow a$ as $n \longrightarrow \infty$.

Since

$$
\begin{aligned}
I_{\Phi}\left(\frac{y_{1}^{(n)}}{a}\right) & =\left(l_{n}-k_{n}\right) \Phi\left(\frac{u_{n}}{a}\right) \\
& \geq \frac{1+1 / n}{a}\left(l_{n}-k_{n}\right) \Phi\left(u_{n}\right) \\
& =\frac{1+1 / n}{a} I_{\Phi}\left(y_{1}^{(n)}\right) \longrightarrow 1
\end{aligned}
$$

that is, $\left\|y_{1}^{(n)}\right\|_{(\Phi)} \longrightarrow a$.

By

$$
\begin{aligned}
& \lim _{\lambda \rightarrow 0} \sup \left\{\frac{I_{\Phi}\left(\lambda y_{m}^{(n)}\right)}{\lambda}: m=1,2, \ldots\right\}=\lim _{\lambda \longrightarrow 0} \frac{I_{\Phi}\left(\lambda x_{n}\right)}{\lambda} \\
& \quad=0
\end{aligned}
$$

we have $y_{m}^{(n)} \stackrel{w}{\rightarrow} 0$ as $m \longrightarrow \infty$.

Therefore

$$
\begin{aligned}
& R W\left(a, l_{(\Phi)}\right) \\
& \quad \geq \sup \left\{\liminf _{m \longrightarrow \infty}\left\|y_{1}^{(n)}+y_{m}^{(n)}\right\|_{(\Phi)}: n=1,2, \ldots\right\} \\
& \quad=1+a,
\end{aligned}
$$

that is to say $R W\left(a, l_{(\Phi)}\right)=1+a$.

Sufficiency. Suppose that there exist $\delta>0$ and $u_{0}>0$ such that $\Phi(u /(1+a)) \leq((1-\delta) /(1+a)) \Phi(u)$ or $\Phi(u / a) \geq$ $((1+\delta) / a) \Phi(u)$ when $|u| \leq u_{0}$. That means there exist $\delta>0$ and $u_{0}>0$ such that $\Phi(u) \leq((1-\delta) /(1+a)) \Phi((1+a) u)$ or $\Phi(u) \leq(a /(1+\delta)) \Phi(u / a)$ when $|u| \leq u_{0}$. It is well known that if $\Phi$ is an Orlicz function, $\lim _{u \longrightarrow 0}(\Phi(u) / u)=b$. If $b>0$, $l_{(\Phi)}$ has Schur property. That is to say, the norm convergence of sequence in $l_{(\Phi)}$ coincides with the weak convergence. In order to discuss the coefficient $R W\left(a, l_{(\Phi)}\right)$ we may assume $b=0$. Put $f(u)=(1+a) \Phi(u) / \Phi((1+a) u)$. By $b=0$, we have $f(u)<1$. Since the function $f$ is continuous in $\left[u_{0}, \Phi^{-1}(1)\right]$, there exists a $u_{1} \in\left[u_{0}, \Phi^{-1}(1)\right]$ such that $f(u) \leq$ $f\left(u_{1}\right)$ for each $u \in\left[u_{0}, \Phi^{-1}(1)\right]$. Suppose $f\left(u_{1}\right)=1$. Then $(1+a) \Phi\left(u_{1}\right) / \Phi\left((1+a) u_{1}\right)=1$ and $\Phi(u)$ is affine function in $\left[0, v_{1}\right]$, where $v_{1}=(1+a) u_{1}$. This contradicts with $b=0$. Therefore $(1+a) \Phi(u) / \Phi((1+a) u)=f\left(u_{1}\right)$. This implies $\Phi(u) \leq\left(f\left(u_{1}\right) /(1+a)\right) \Phi((1+a) u)$ when $u \in\left[u_{0}, \Phi^{-1}(1)\right]$. It is shown that there exist $\delta>0$ and $u_{0}>0$ such that $\Phi(u) \leq$ $((1-\delta) /(1+a)) \Phi((1+a) u)$ or $\Phi(u) \leq(a /(1+\delta)) \Phi(u / a)$ when $|u| \leq \Phi^{-1}(1)$. Using $\Phi \in \delta_{2}$ there exists a $\beta>0$ such that $I_{\Phi}(x) \geq \beta$ when $\|x\|_{(\Phi)}=a$.

Put

$$
B_{a}=\left\{u \in\left[0, \Phi^{-1}(1)\right]: \Phi\left(\frac{u}{a}\right) \geq \frac{1+\delta}{a} \Phi(u)\right\}
$$


and

$$
\begin{aligned}
& B_{1+a} \\
& \quad=\left\{u \in\left[0, \Phi^{-1}(1)\right]: \Phi\left(\frac{u}{1+a}\right) \geq \frac{1-\delta}{1+a} \Phi(u)\right\} .
\end{aligned}
$$

Let $N_{a}=\left\{i: x(i) \in B_{a}\right\}$ and $N_{1+a}=\left\{i: x(i) \in B_{1+a}\right\}$.

We consider the following two cases.

Case I $\left(\sum_{i \in N_{a}} \Phi(x(i)) \geq \beta / 2\right)$. In this case, from

$$
\begin{aligned}
1 & \geq I_{\Phi}\left(\frac{x}{a}\right)=\sum_{i \in N_{a}} \Phi\left(\frac{x(i)}{a}\right)+\sum_{i \in N_{1+a}} \Phi\left(\frac{x(i)}{a}\right) \\
& \geq \frac{1+\delta}{a} \sum_{i \in N_{a}} \Phi(x(i))+\frac{1}{a} \sum_{i \in N_{1+a}} \Phi(x(i)) \\
& =\frac{1}{a} I_{\Phi}(x)+\frac{\delta \beta}{2 a},
\end{aligned}
$$

we get

$$
I_{\Phi}(x) \leq a-\frac{\delta \beta}{2 a} .
$$

For any $y \in B\left(l_{\Phi}\right)$ with $\operatorname{supp}(y)=\{i \in N: y(i) \neq 0\} \cap$ $\operatorname{supp}(x)=\emptyset$, the following inequality holds:

$$
I_{\Phi}(x+y)=I_{\Phi}(x)+I_{\Phi}(y) \leq a-\frac{\delta \beta}{2 a}+1 .
$$

Therefore $\|x+y\|_{(\Phi)} \leq I_{\Phi}(x+y) \leq a-\delta \beta / 2 a+1$, that is to say $R W\left(a, l_{(\Phi)}\right) \leq a-\delta \beta / 2 a+1<1+a$.

Case II $\left(\sum_{i \in N_{1}+a} \Phi(x(i)) \geq \beta / 2\right)$. In this case, for any $y \in B\left(l_{\Phi}\right)$ with $\operatorname{supp}(y)=\{i \in N: y(i) \neq 0\} \cap \operatorname{supp}(x)=\emptyset$, the following inequality holds:

$$
\begin{aligned}
I_{\Phi}\left(\frac{x+y}{1+a}\right)= & I_{\Phi}\left(\frac{x}{1+a}\right)+I_{\Phi}\left(\frac{y}{1+a}\right) \\
\leq & \sum_{i \in N_{a}} \Phi\left(\frac{x(i)}{1+a}\right)+\sum_{i \in N_{1+a}} \Phi\left(\frac{x(i)}{1+a}\right) \\
& +\frac{1}{1+a} I_{\Phi}(y) \\
\leq & \frac{1}{1+a} \sum_{i \in N_{a}} \Phi(x(i)) \\
& +\frac{1-\delta}{1+a} \sum_{i \in N_{1+a}} \Phi(x(i))+\frac{1}{1+a} \\
= & \frac{1}{1+a} I_{\Phi}(x)+\frac{1}{1+a}-\frac{\delta \beta}{2(1+a)} .
\end{aligned}
$$

From $I_{\Phi}(x) \leq\|x\|_{(\Phi)}=a \leq 1$, we have

$$
I_{\Phi}\left(\frac{x+y}{1+a}\right) \leq 1-\frac{\delta \beta}{2(1+a)},
$$

that is to say

$$
\|x+y\|_{(\Phi)} \leq \frac{2+a-\delta \beta}{2}
$$

Therefore $R W\left(a, l_{(\Phi)}\right) \leq(2+a-\delta \beta) / 2<1+a$.

Theorem 17. For $a \in(0,1), R\left(a, l_{(\Phi)}\right)<1+a$ if and only if $\Phi \in \delta_{2}$.

Proof. It is easy to get the following formula:

$$
\begin{aligned}
& R(a, X)=\sup \left\{\liminf _{n \longrightarrow \infty}\left\|x_{n}+x\right\|_{(\Phi)}: x_{n} \stackrel{w}{\rightarrow} 0,\left\{x_{n}, x\right\}\right. \\
& \left.\subset B(X),\left\|x_{n}-x_{m}\right\|_{(\Phi)} \leq 1\right\} .
\end{aligned}
$$

Take any weak null sequence $\left\{x_{n}\right\} \quad \subset \quad B\left(l_{(\Phi)}\right)$ with $\| x_{n}-$ $x_{m} \|_{(\Phi)} \leq 1$. Hence $I_{\Phi}\left(x_{n}-x_{m}\right) \leq 1$. Using $x_{n} \stackrel{w}{\rightarrow} 0$, for any $\varepsilon \in(0,1 / 2)$ and subsequence $\left\{x_{n_{i}}\right\}$ of $\left\{x_{n}\right\}$ such that $I_{\Phi}\left(x_{n_{i}}\right) \leq 1 / 2+\varepsilon$. By $\Phi \in \delta_{2}$, there exists $\delta \in(0,1)$ such that $\left\|x_{n_{i}}\right\|_{(\Phi)} \leq \delta$. Therefore for any $x \in l_{\Phi},\|x\|_{(\Phi)}=a$. It holds

$$
\left\|x_{n_{i}}+x\right\|_{(\Phi)} \leq\left\|x_{n_{i}}\right\|_{(\Phi)}+\|x\|_{(\Phi)} \leq \delta+a<1+a
$$

\section{Data Availability}

No data were used to support this study.

\section{Conflicts of Interest}

The authors declare that they have no conflicts of interest.

\section{Acknowledgments}

This research is supported by NSF of China (Grant No. (11871181)).

\section{References}

[1] W. A. Kirk, "A fixed point theorem for mappings which do not increase distances," The American Mathematical Monthly, vol. 72, pp. 1004-1006, 1965.

[2] S. Reich, "The fixed point property for nonexpansive mappings, I, II," The American Mathematical Monthly, vol. 83, pp. 266-268, 1976.

[3] K. Goebel and S. Reich, Uniform Convexity, Hyperbolic Geometry, and Nonexpansive Mappings, vol. 83 of Monographs and Textbooks in Pure and Applied Mathematics, Marcel Dekker, New York, NY, USA, 1984.

[4] S. Prus, "Nearly uniformly smooth Banach spaces," Bollettino della Unione Matematica Italiana, vol. 10, no. 7, pp. 507-521, 1989.

[5] J. G. Falset, "The fixed point property in Banach spaces with the NUS-property," Journal of Mathematical Analysis and Applications, vol. 1997, no. 215, pp. 532-542, 1997. 
[6] S. Prus, "Banach spaces with the uniform opial property," Nonlinear Analysis: Theory, Methods \& Applications, vol. 18, no. 8, pp. 697-704, 1992.

[7] T. Dominguez Benavides, "A geometrical coefficient implying the fixed point property and stability results," Houston Journal of Mathematics, vol. 22, no. 4, pp. 835-849, 1996.

[8] T. D. Benavides, "Modulus of nearly uniform smoothness and Lindenstrauss formulae," Glasgow Mathematical Journal, vol. 37, no. 2, pp. 143-153, 1995.

[9] J. JesúsGarcía-False, E. Llorens-Fuster, and E. M. MazcunanNavarro, "Uniformly nonsquare Banach spaces have the fixed point property for nonexpansive mappings," Journal of Functional Analysis, vol. 233, no. 2, pp. 494-514, 2006.

[10] J. G. Falset, "The fixed point property in Banach spaces with the NUS-property," Journal of Mathematical Analysis and Applications, vol. 215, no. 2, pp. 532-542, 1997.

[11] J. Diestel, Sequence and Series in Banach Spaces, vol. 92 of Graduate Texts in Mathematics, Springer-Verlag, Berlin, Germany, 1984.

[12] L. V. Kantorovich and G. P. Akilov, Functional analysis, Nauka Moscow, 1983.

[13] S. Chen, Geometry of Orlicz Spaces, Dissertations Math, Warszawa, Poland, 1996.

[14] J. Musielak, Orlicz Spaces and Modular Spaces, vol. 1034 of Lecture Notes in Mathematics, Springer, Berlin, Germany, 1983.

[15] M. M. Rao and Z. D. Ren, Theory of Orlicz Spaces, Marcel Dekker, New York, NY, USA, 1991.

[16] H. Hudzik and M. Mastylo, "Almost isometric copies of lo in some banach spaces," Proceedings of the American Mathematical Society, vol. 119, no. 1, pp. 209-205, 1993.

[17] Y. Cui and H. Hudzik, "On the García-Falset coefficient in Orlicz sequence spaces equipped with the Orlicz norm," in Function Spaces and Applications, pp. 60-68, Narosa Publishing House, New Delhi, India, 2000. 


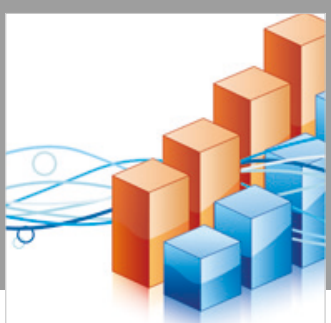

Advances in

Operations Research

\section{-n-m}
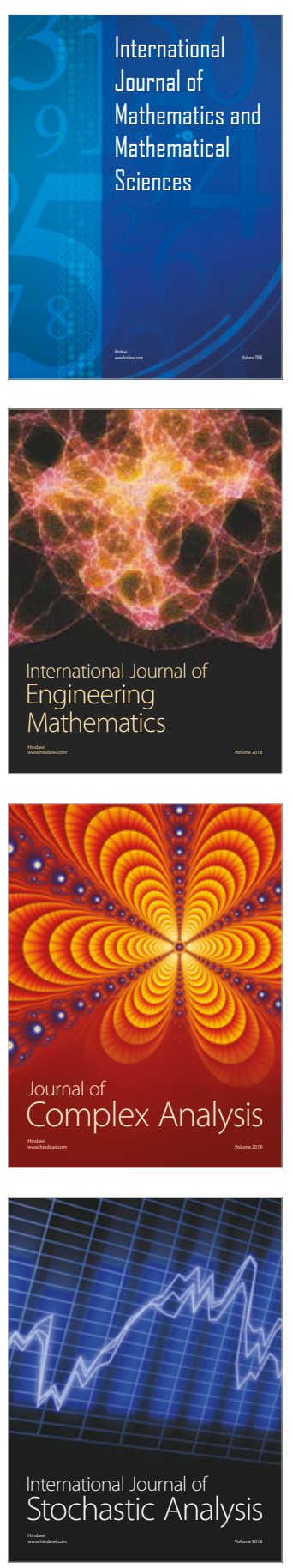
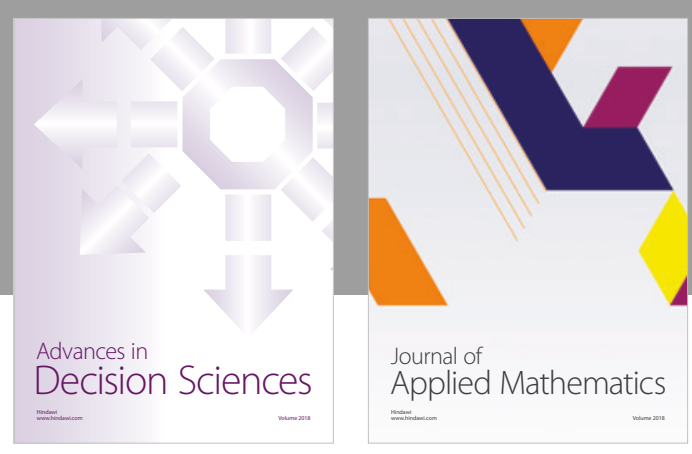

Journal of

Applied Mathematics
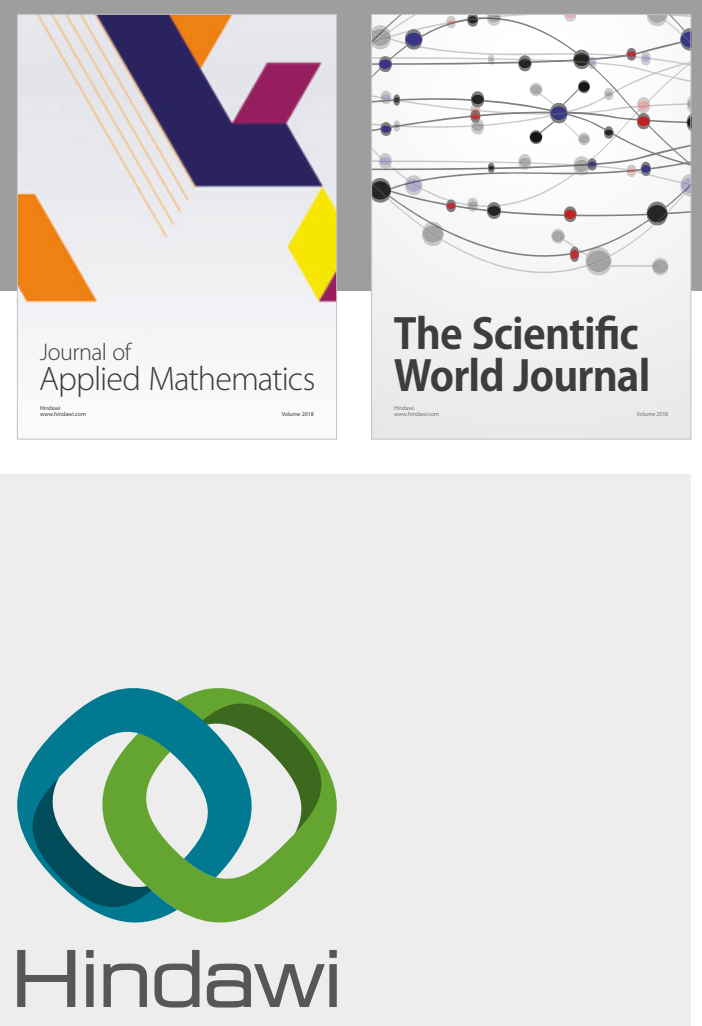

Submit your manuscripts at

www.hindawi.com

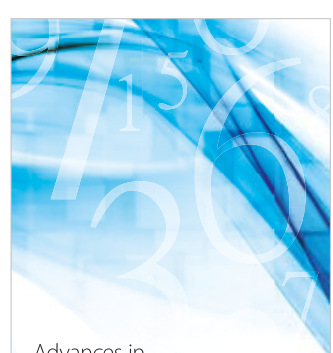

Advances in
Numerical Analysis
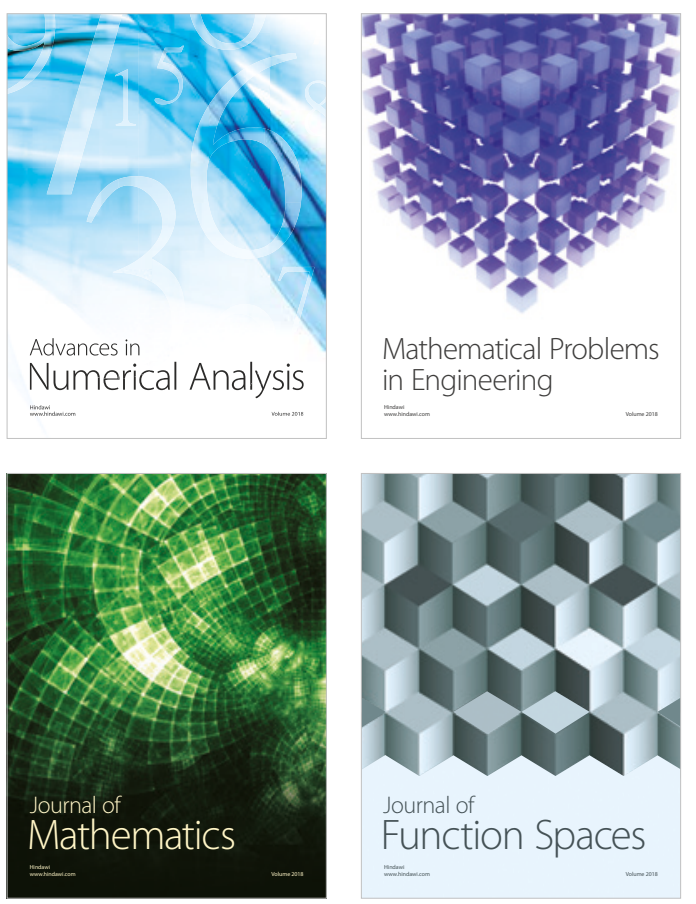

Mathematical Problems in Engineering

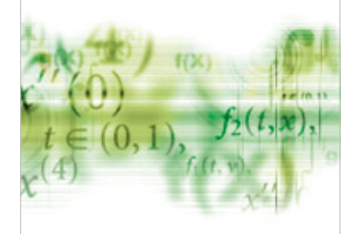

International Journal of

Differential Equations

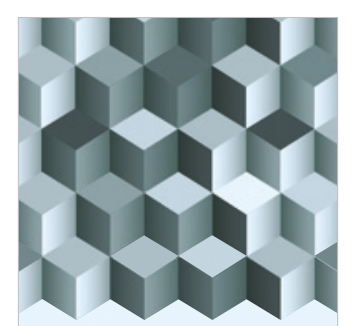

Journal of

Function Spaces

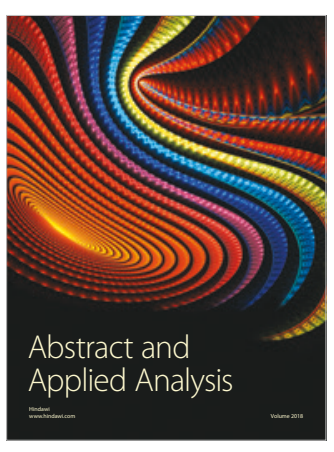

The Scientific

World Journal

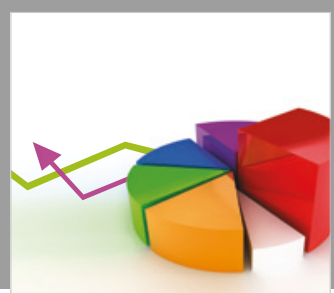

Journal of

Probability and Statistics
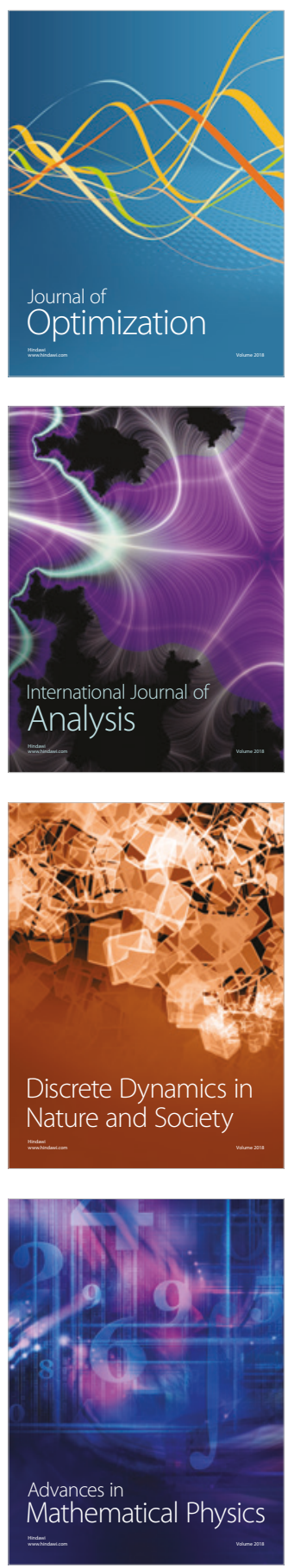